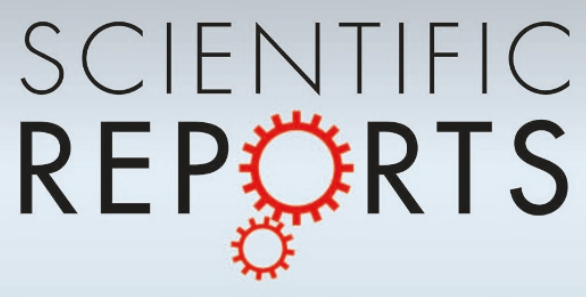

OPEN

SUBJECT AREAS:

GENETIC ENGINEERING

INDUSTRIAL MICROBIOLOGY

Received

28 October 2014

Accepted

11 February 2015

Published

12 March 2015

Correspondence and requests for materials should be addressed to

C.G. (jieerbu@sdu. edu.cn)

* Current address: School of Life Sciences

\& Biotechnology, Shanghai Jiao Tong University, Shanghai 200240, People's Republic of China.

\section{Production of diacetyl by metabolically engineered Enterobacter cloacae}

\author{
Lijie Zhang, Yingxin Zhang, Qiuyuan Liu, Liying Meng, Mandong Hu, Min Lv, Kun Li, Chao Gao, Ping Xu* \\ \& Cuiqing $\mathrm{Ma}$
}

State Key Laboratory of Microbial Technology, Shandong University, Jinan 250100, People's Republic of China.

Diacetyl, a high value product that can be extensively used as a food ingredient, could be produced from the non-enzymatic oxidative decarboxylation of $\alpha$-acetolactate during 2,3-butanediol fermentation. In this study, the 2,3-butanediol biosynthetic pathway in Enterobacter cloacae subsp. dissolvens strain SDM, a good candidate for microbial 2,3-butanediol production, was reconstructed for diacetyl production. To enhance the accumulation of the precursor of diacetyl, the $\alpha$-acetolactate decarboxylase encoding gene ( $b u d A)$ was knocked out in strain SDM. Subsequently, the two diacetyl reductases DR-I (gdh) and DR-II (budC) encoding genes were inactivated in strain SDM individually or in combination to decrease the reduction of diacetyl. Although the engineered strain E. cloacae SDM ( $\Delta$ budA $\Delta b u d C)$ was found to have a good ability for diacetyl production, more $\alpha$-acetolactate than diacetyl was produced simultaneously. In order to enhance the nonenzymatic oxidative decarboxylation of $\alpha$-acetolactate to diacetyl, $20 \mathrm{mM} \mathrm{Fe}^{3+}$ was added to the fermentation broth at the optimal time. In the end, by using the metabolically engineered strain $E$. cloacae SDM ( $\Delta$ budA $\Delta$ budC), diacetyl at a concentration of $1.45 \mathrm{~g} / \mathrm{L}$ was obtained with a high productivity $(0.13 \mathrm{~g} /(\mathrm{L} \cdot \mathrm{h}))$. The method developed here may be a promising process for biotechnological production of diacetyl.

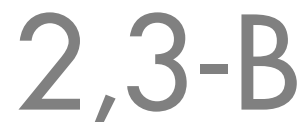

utanediol (2,3-BD) can be efficiently produced by microbial fermentation as a platform and fuel bio-chemical ${ }^{1-4}$. Many microorganisms such as Enterobacter, Klebsiella, Bacillus, and Serratia could be used to produce 2,3-BD from biomass ${ }^{5-10}$. Three key enzymes, including $\alpha$-acetolactate synthase (ALS), $\alpha$-acetolactate decarboxylase (ALDC), and 2,3-butanediol dehydrogenase $(\mathrm{BDH})$, are involved in the biosynthesis of 2,3-BD from pyruvate ${ }^{2,4}$. Two molecules of pyruvate are condensed to $\alpha$-acetolactate by ALS. Then, ALDC catalyzes the decarboxylation of $\alpha$-acetolactate to produce (3R)-acetoin $((3 R)-\mathrm{AC}) .(3 R)$-AC will be reduced to meso-2,3-BD and $(2 R, 3 R)-2,3-\mathrm{BD}$ by meso-2,3-BDH and $(2 R, 3 R)-2,3-$ $\mathrm{BDH}$, respectively ${ }^{11}$. Since meso-2,3-BDH and $(2 R, 3 R)-2,3-\mathrm{BDH}$ could also catalyze the reduction of diacetyl to produce $\mathrm{AC}$ and 2,3-BD, these enzymes were also called as diacetyl reductase (DR).

$\alpha$-Acetolactate produced in the 2,3-BD fermentation is unstable and can also be catalyzed through nonenzymatic oxidative decarboxylation to produce diacety ${ }^{12,13}$. Diacetyl is an important flavor compound responsible for the buttery aroma of many dairy products, and is used as an ingredient in the food industry ${ }^{14}$. Diacetyl could also be produced through chemical methods. However, microbial production of diacetyl is preferred over chemical synthesis as a food or perfume additive due to the safety reasons ${ }^{15}$.

Various microorganisms such as lactic acid bacteria, Candida glabrata and Enterobacter aerogenes, have been used in the production of diacety ${ }^{12,16}$. For instance, a Lactococcus lactis mutant with low ALDC and lactate dehydrogenase activities was able to overproduce diacetyl with a final concentration of $0.52 \mathrm{~g} / \mathrm{L}^{17}$. Studies by Guo et al. demonstrated that overexpression of NADH oxidase could efficiently tune the lactate and diacetyl production in L. lactis $^{18}$. A combination of NADH oxidase overexpression with ALDC inactivation in L. lactis could produce diacetyl with a yield of $0.16 \mathrm{~mol} / \mathrm{mol}$ of glucose, which was the highest yield of diacetyl synthesis till now $^{14}$. Recently, C. glabrata CCTCC M202019 was metabolically engineered for diacetyl production. A high titer of $4.7 \mathrm{~g} / \mathrm{L}$ of diacetyl was achieved with a yield of $0.10 \mathrm{~mol} / \mathrm{mol}$ and a productivity of $0.07 \mathrm{~g} /(\mathrm{L} \cdot \mathrm{h})^{12}$. Diacetyl is a byproduct of the 2,3-BD fermentation, but most of the studies mentioned above have been based on strains with low 2,3-butanediol producing capabilities ${ }^{14,19-20}$. Due to their low efficiency of the glycolytic flux to diacetyl, the productivity and yield of diacetyl using these strains should be further enhanced for industrial production.

In our previous reports, Enterobacter cloacae subsp. dissolvens SDM can efficiently produce 2,3-BD with a high productivity and a high yield 5 . The key enzymes for 2,3-BD metabolism, including ALS, ALDC, and the two DRs, were annotated based on the genome sequence of the strain ${ }^{21}$. Thus, it might be a good candidate for production of 
diacetyl through metabolic engineering. In the present work, the ALDC encoding gene budA has been knocked out and the DRs encoding genes were also inactivated to construct a diacetyl producer (Figure 1 ). $\mathrm{Fe}^{3+}$ was added to the medium to improve the nonenzymatic oxidative decarboxylation of $\alpha$-acetolactate to produce diacetyl. Through the metabolic engineering approach described, $1.45 \mathrm{~g} / \mathrm{L}$ diacetyl was synthesized within $11.3 \mathrm{~h}$ with a high yield of $0.21 \mathrm{~mol} /$ mol using glucose as substrate.

\section{Results}

Potential for diacetyl production by E. cloacae SDM. 2,3-BD exists in three stereoisomeric forms: $(2 R, 3 R)-2,3-\mathrm{BD},(2 S, 3 S)-2,3-\mathrm{BD}$, and meso-2,3-BD. Recently, the mechanism of 2,3-BD stereoisomer formation was identified in 2,3-BD producing strains including $K$. pneumonia. $(2 R, 3 R)-2,3-\mathrm{BD}$ and meso-2,3-BD are mainly produced from the $(3 R)-\mathrm{AC}$ by the reactions catalyzed by $(2 R, 3 R)-2,3-\mathrm{BDH}$ and meso-2,3-BDH, respectively ${ }^{11}$. $(2 S, 3 S)-2,3-\mathrm{BD}$ could only be produced by the meso-2,3-BDH catalyzed reduction of $(3 S)-\mathrm{AC}$, which is not an enzymatic decarboxylation product of $\alpha$-acetolactate but a reduction product of diacetyl ${ }^{11}$. As shown in Figure 2 I-B, the main metabolic products of E. cloacae SDM were meso-2,3-BD and

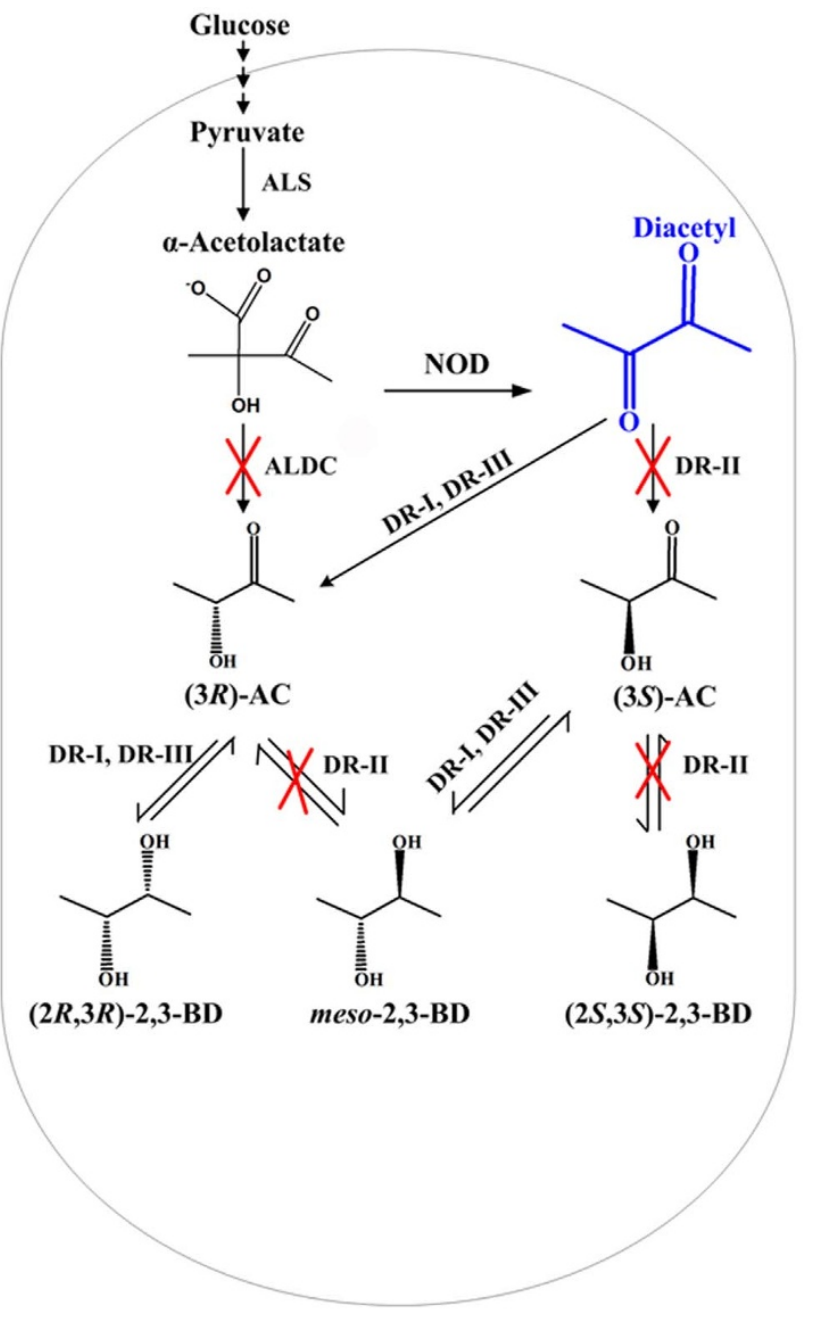

Figure $1 \mid$ Engineered pathway for diacetyl production in E. cloacaeSDM. NOD, nonenzymatic oxidative decarboxylation; ALS, $\alpha$-acetolactate synthase, encoded by budB; ALDC, $\alpha$-acetolactate decarboxylase, encoded by budA; DR-I, diacetyl reductase-I (glycerol dehydrogenase), encoded by $g d h$; DR-II, diacetyl reductase-II (meso-2,3-butanediol dehydrogenase), encoded by budC; DR-III, an undiscovered diacetyl reductase. Crosses represent the enzyme inactivation performed in this study.
(2S,3S)-2,3-BD when glucose was used as the carbon source. Low concentrations of $(3 R)$-AC and $(3 S)$-AC were also produced under aerobic conditions. $(2 S, 3 S)-2,3-\mathrm{BD}$ could only be produced via the meso-2,3-BDH catalyzed two step reduction of diacetyl, the nonenzymatic oxidative decarboxylation product of $\alpha$-acetolactate. Thus, diacetyl was produced as an intermediate of 2,3-BD biosynthesis in E. cloacae SDM. Redirecting more carbon flux toward the 2,3-BD into diacetyl through metabolic engineering of E. cloacae SDM might result in an efficient strain for the production of diacetyl.

Metabolic characteristics of the ALDC mutant of $E$. cloacae SDM. Although there was nonenzymatic oxidative decarboxylation of $\alpha$ acetolactate in E. cloacae SDM, little diacetyl accumulated in the 2,3-BD fermentation process (Figure 2 I-A Figure 2 I-C and Table 1). Since $\alpha$-acetolactate is mainly subjected to enzymatic conversion to $(3 R)$-AC catalyzed by ALDC besides nonenzymatic oxidative conversion to diacetyl, lack of diacetyl accumulation might be due to the high degradation rate of $\alpha$-acetolactate. Since the enzymatic irreversible reaction drains the available pool of $\alpha$ acetolactate for diacetyl formation, knockout of the ALDC might be an effective method for the enhancement of diacetyl production in the E. cloacae strain SDM.

In this study, E. cloacae SDM ( $\triangle b u d A)$ was constructed by knockout of the budA gene (Gene bank: 13167655) through allele exchange (Figure 2 II-A). The effects of budA gene deletion on the ALDC activity and diacetyl formation of strain SDM are shown in Table 1 and Figure 2 II-C, respectively. In the native strain, the ALDC activity towards $\alpha$-acetolactate was $3.81 \pm 0.16 \mathrm{U} / \mathrm{mg}$ while little ALDC activity $(0.04 \pm 0.00 \mathrm{U} / \mathrm{mg})$ was detected in E. cloacae SDM $(\triangle b u d A)$. After $36 \mathrm{~h}$ fermentation, the concentration of diacetyl produced by E. cloacae SDM ( $\Delta b u d A)$ was $59.7 \mathrm{mg} / \mathrm{L}$ while only $2.85 \mathrm{mg} / \mathrm{L}$ diacetyl was obtained by the native strain SDM (Table 1). Besides diacetyl, (3R)-AC, (3S)-AC, (2R,3R)-2,3-BD, (2S,3S)-2,3-BD, and meso-2,3-BD were also detected in the medium (Figure 2 II-B). These results indicate that the diacetyl would also be converted into those compounds in E. cloacae SDM ( $\Delta b u d A)$.

Inactivation of DR-I in the ALDC mutant of E. cloacae SDM. Glycerol dehydrogenase (GDH) belongs to the medium-chain dehydrogenase family and accepts a broad range of substrates. Diacetyl could be reduced to $(3 R)-\mathrm{AC}$ and $(2 R, 3 R)-2,3-\mathrm{BD}$ by the GDH in $K$. pneumonia. A gdh gene (Gene bank: 13166340), which exhibits $59 \%$ sequence identity with that of K. pneumonia, was identified in the genome sequence of E. cloacae SDM. In this study, the protein encoded by $g d h$ gene was renamed as DR-I due to its diacetyl reduction activity. As shown in Table 1, inactivation of DR-I would result in a lower DR activity of E. cloacae SDM $(\Delta b u d A \Delta g d h)$ than that of the strain E. cloacae SDM and the mutant strain E. cloacae SDM $(\triangle b u d A)$. However, the concentration of diacetyl increased modestly to only $326.7 \mathrm{mg} / \mathrm{L}$ (Table 1 ). (3R)-AC, (3S)-AC, $(2 R, 3 R)-2,3-\mathrm{BD}$, $(2 S, 3 S)-2,3-\mathrm{BD}$, and meso-2,3-BD would still accumulate during the fermentation (Figure 2 III-B).

Inactivation of DR-II in the ALDC mutant of E. cloacae SDM. The genes that encode ALDC, ALS, and meso-2,3-BDH are sequentially clustered in one operon in E. cloacae SDM (Figure S1). Our previously studied enzymatic reactions showed that meso-2,3-BDH can catalyze the conversion of diacetyl to (3S)-AC and further to $(2 S, 3 S)-2,3-\mathrm{BD}$ as well as $(3 R)-\mathrm{AC}$ to meso-2,3-BD. In this study, the meso-2,3-BDH (renamed as DR-II) encoding gene budC (Gene bank: 13167657) was knocked out through the allele exchange in $E$. cloacae SDM ( $\triangle b u d A)$ (Figure 2 IV-A).

As shown in Table 1, inactivation of DR-II would result in a sharp decrease of DR activity in E. cloacae SDM $(\Delta b u d A \Delta b u d C)$. The concentration of diacetyl increased to $416.1 \mathrm{mg} / \mathrm{L}$ after $36 \mathrm{~h}$ fermentation (Figure $2 \mathrm{IV}-\mathrm{C}$, Table 1 ). The budC mutant lost the ability to produce $(2 S, 3 S)-2,3-\mathrm{BD}$ and meso-2,3-BD (Figure 2 IV-B). This 
I

A

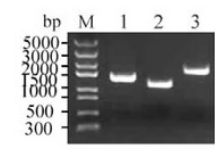

C

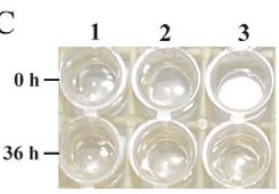

III

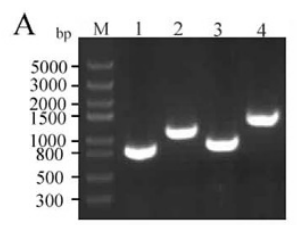

C

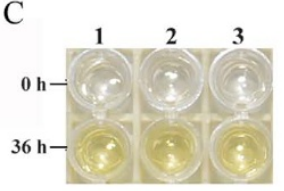

B

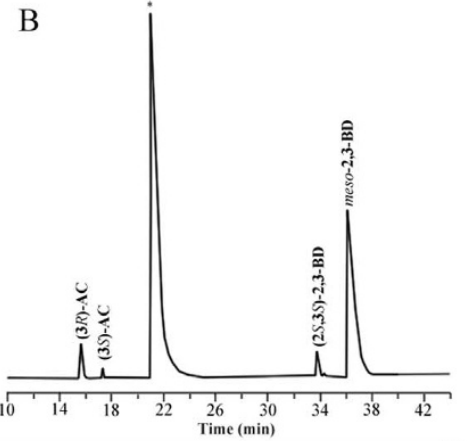

IV

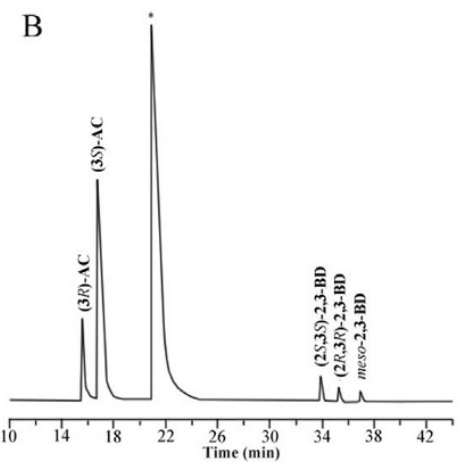

II
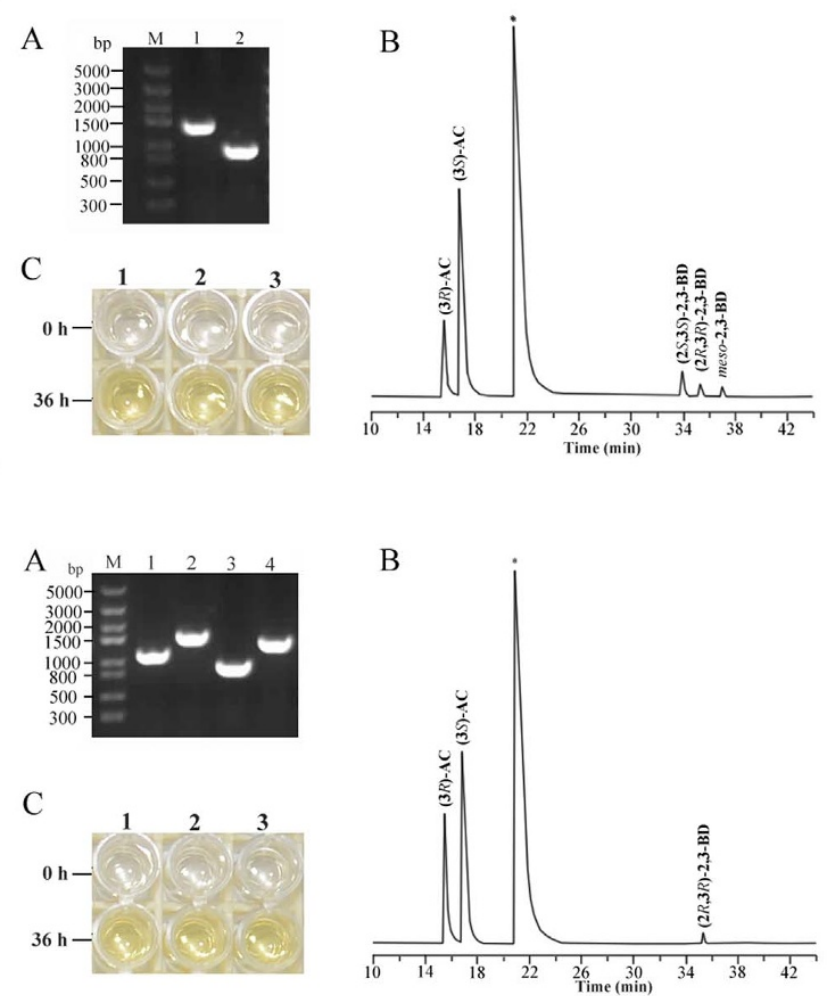

Figure $2 \mid$ Molecular authentication and metabolic production analysis of E. cloacae SDM and its derivatives. Panel I: E. cloacae SDM; Panel II: SDM $(\Delta b u d A)$; Panel III: SDM ( $\Delta b u d A \Delta g d h)$; Panel IV: SDM $(\Delta b u d A \Delta b u d C)$. (A): Analysis of PCR fragments to confirm disruption of the gene. Lane M, molecular mass standard (Trans5K). (B): Fermentation products identified by GC. (C): Colorimetric detection of diacetyl (1,2, 3 means all assays were performed by triplicate cultures). I-A: Lane 1-3: $b u d A, g d h, b u d C$ products amplified with SDM genomic DNAs as the template. II-A: Lane 1-2, budA products amplified with SDM and SDM $(\triangle b u d A)$ genomic DNAs as the templates, respectively. III-A: lane 1-2: gdh products amplified with SDM $(\Delta b u d A \Delta g d h)$ and SDM genomic DNAs as the templates, respectively; lane 3-4: budA products amplified with SDM ( $\Delta b u d A \Delta g d h)$ and SDM genomic DNAs as the templates, respectively. IV-A: Lane 1-2: budC products amplified with SDM $(\Delta b u d A \Delta b u d C)$ and SDM genomic DNAs as the templates, respectively; lane 3-4: budA products amplified with SDM ( $\Delta b u d A \Delta b u d C)$ and SDM genomic DNAs as the templates, respectively.

phenotype indicates that the formation of both $(2 S, 3 S)-2,3-\mathrm{BD}$ and meso-2,3-BD depends on the activity of DR-II.

Then, DR-I and DR-II were both inactivated in the ALDC mutant of E. cloacae SDM (Figure S2). As shown in Table 1, the DR activity would further decrease in the DR-I and DR-II double mutant. However, the glucose consumed, biomass, and concentration of diacetyl would also decrease in the mutant of E. cloacae SDM ( $\Delta b u d A \Delta b u d C \Delta g d h)$. Since the concentration $(416.10 \mathrm{mg} / \mathrm{L})$ of diacetyl obtained by E. cloacae SDM $(\Delta b u d A \Delta b u d C)$ was higher than that of other strains, E. cloacae SDM ( $\Delta b u d A \Delta b u d C)$ was chosen for further investigation.

Diacetyl production by $E$. cloacae SDM ( $\Delta$ budA $\Delta$ budC). Diacetyl production using E. cloacae SDM ( $\Delta b u d A \Delta b u d C)$ was conducted at $37^{\circ} \mathrm{C}$ in $300-\mathrm{mL}$ shake flasks containing $50 \mathrm{~mL}$ medium. The medium was M9 medium supplemented with $18 \mathrm{~g} / \mathrm{L}$ glucose and $5 \mathrm{~g} / \mathrm{L}$ yeast extract ${ }^{22}$. The initial $\mathrm{pH}$ was 7.4. As shown in Figure 3, $59.8 \mathrm{mg} / \mathrm{L}$ diacetyl was obtained from $15 \mathrm{~g} / \mathrm{L}$ glucose after $12 \mathrm{~h}$ of bioconversion. The yield of diacetyl was only at $0.83 \%$ of the theoretical value.

The concentration of $\alpha$-acetolactate produced by $E$. cloacae SDM ( $\triangle b u d A \Delta b u d C)$ was also analyzed during the $12 \mathrm{~h}$ of bioconversion. $\alpha$-Acetolactate of $2.94 \mathrm{~g} / \mathrm{L}$ was produced. This indicated that the strain E. cloacae SDM ( $\Delta b u d A \Delta b u d C)$ showed an almost $32: 1$ $(\mathrm{mol} / \mathrm{mol})$ co-production of $\alpha$-acetolactate and diacetyl. Thus, diacetyl production could be further enhanced by the transformation of $\alpha$-acetolactate accumulated in medium.

Optimization of the addition time of $\mathrm{Fe}^{3+}$. In order to achieve higher diacetyl production, non-enzymatic oxidative decarboxyla-

Table 1 | Enzyme activities, glucose consumption and diacetyl production of E. cloacae SDM and its derivatives ${ }^{a}$

\begin{tabular}{|c|c|c|c|c|}
\hline Strain & $\mathrm{DR}(\mathrm{U} / \mathrm{mg})$ & $\mathrm{ALDC}(\mathrm{U} / \mathrm{mg})$ & Glucose (g/L) & Diacetyl (mg/L) \\
\hline SDM & $14.20 \pm 1.11$ & $3.81 \pm 0.16$ & 35 & $2.85 \pm 1.57$ \\
\hline $\operatorname{SDM}(\Delta b u d A)$ & $5.72 \pm 0.23$ & $0.04 \pm 0.00$ & 19.5 & $59.70 \pm 11.24$ \\
\hline SDM $(\Delta b u d A \Delta g d h)$ & $5.29 \pm 0.13$ & $0.003 \pm 0.00$ & 17 & $326.66 \pm 7.54$ \\
\hline SDM ( $\Delta$ budA $\Delta$ budC $\Delta g d h)$ & $0.47 \pm 0.04$ & $0.003 \pm 0.00$ & 14.5 & $318.31 \pm 33.08$ \\
\hline
\end{tabular}

DR: diacetyl reductase; ALDC: $\alpha$-acetolactate decarboxylase.

aData are the means \pm standard deviations (SDs) from three parallel experiments. 


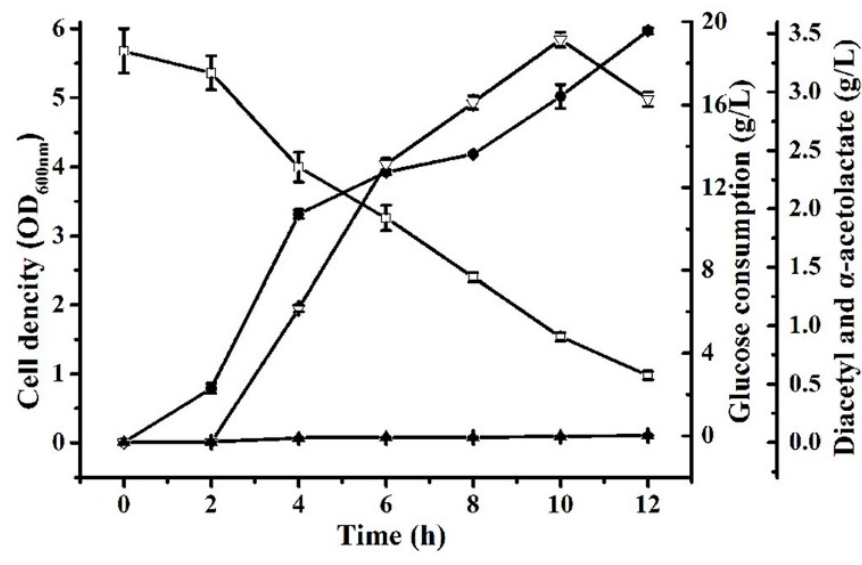

Figure 3 Diacetyl production from glucose using E. cloacae SDM ( $\Delta$ budA $\Delta$ budC). Glucose $(\square)$, Cell density $(\bullet)$, $\alpha$-Acetolactate $(\nabla)$, Diacetyl $(\boldsymbol{\Delta})$. The bioconversion was carried out at $37^{\circ} \mathrm{C}$ in $300-\mathrm{mL}$ shake flasks containing $50 \mathrm{~mL}$ of medium with $\mathrm{pH}$ adjusted to 7.4. The initial glucose concentration used was $18 \mathrm{~g} / \mathrm{L}$ approximately. Error bars indicate standard deviation $(\mathrm{n}=3)$.

tion of $\alpha$-acetolactate should be enhanced. It was reported that $\alpha$ acetolactate could be further converted to diacetyl by addition of $\mathrm{Fe}^{3+12,23}$. To study the effect of the addition time of $\mathrm{Fe}^{3+}$ on diacetyl production, $20 \mathrm{mM} \mathrm{Fe}^{3+}$ was added at $0,2,4,6,8,10$, and $12 \mathrm{~h}$, respectively. The diacetyl production and glucose consumption were detected after $14 \mathrm{~h}$ fermentation.

As shown in Figure 4A and Figure 4B, although addition of $\mathrm{Fe}^{3+}$ at $12 \mathrm{~h}$ would result in a higher glucose utilization, the highest diacetyl concentration of $1.37 \mathrm{~g} / \mathrm{L}$ was acquired when $20 \mathrm{mM} \mathrm{Fe}^{3+}$ was added at $10 \mathrm{~h}$. Addition of $\mathrm{Fe}^{3+}$ at the beginning of the fermentation would inhibit the utilization of glucose and thus decrease the production of diacetyl by E. cloacae SDM ( $\Delta b u d A \Delta b u d C)$.

Batch bioconversion under optimal conditions. Combining the results mentioned above, an optimal system for the production of diacetyl using E. cloacae SDM ( $\Delta b u d A \Delta b u d C)$ was developed. Bioconversion was firstly conducted under the conditions mentioned above for $10 \mathrm{~h}$ (Figure $5 \mathrm{~A}$ ). Then, $20 \mathrm{mM} \mathrm{Fe}^{3+}$ was added in the fermentation medium. As shown in Figure 5B, $1.45 \mathrm{~g} / \mathrm{L}$ diacetyl was produced in $80 \mathrm{~min}$ after the addition of $20 \mathrm{mM} \mathrm{Fe}^{3+}$. Glucose of $14.8 \mathrm{~g} / \mathrm{L}$ was consumed during the bioconversion process. The yield of diacetyl was $0.21 \mathrm{~mol} / \mathrm{mol}$ glucose. During the two-step bioconversion process, diacetyl was produced with a high productivity of $0.13 \mathrm{~g} /(\mathrm{L} \cdot \mathrm{h})$.

\section{Discussion}

Diacetyl has a strong buttery flavor and is mainly existed at low concentration in many dairy products, such as butter, beer, and fresh cheeses. Its formation in dairy products mainly results from the catabolism of $\alpha$-acetolactate during 2,3-BD fermentation by certain species of lactic acid bacteria ${ }^{14}$. Due to the excellent performance of $E$. cloacae SDM as an efficient 2,3-BD producing strain, developing a metabolically engineered strain based on E. cloacae SDM through redirecting carbon flux toward the 2,3-BD pathways for the production of diacetyl is quite attractive and promising.

In the present study, the diacetyl production from glucose by $E$. cloacae SDM was firstly conducted through two genetic strategies: (i) inactivation of the ALDC gene ( $b u d A)$ to avoid enzymatic conversion of the diacetyl precursor $\alpha$-acetolactate to (3R)-AC as described previously ${ }^{14}$ and (ii) inactivation of the DR gene to avoid enzymatic reduction of diacetyl. Two DRs encoding genes ( $g d h$ and $b u d C$ ) were identified in the genome sequence of E. cloacae SDM. E. cloacae SDM $(\Delta b u d A \Delta b u d C \Delta g d h)$ produced diacetyl at a concentration (318.31 mg/L) lower than that of E. cloacae SDM ( $\Delta b u d A \Delta b u d C)$ $(416.10 \mathrm{mg} / \mathrm{L})$. This result indicates that DR might be important to strain SDM for glucose utilization and cell growth. On the other hand, when DR-I and DR-II were both inactivated in the ALDC mutant, (3R)-AC, (3S)-AC, and $(2 R, 3 R)-2,3-\mathrm{BD}$ could still be detected (Figure S2), indicating the presence of the third DR (DRIII) responsible for these chemical production in E. cloacae strain SDM (Figure 1).

Although $2.94 \mathrm{~g} / \mathrm{L} \alpha$-acetolactate was produced from $15 \mathrm{~g} / \mathrm{L}$ glucose after $12 \mathrm{~h}$ of bioconversion, only $59.8 \mathrm{mg} / \mathrm{L}$ diacetyl was obtained and the final molar ratio of $\alpha$-acetolactate and diacetyl was 32:1 (Figure 3), implying an inefficient NOD of $\alpha$-acetolactate to diacetyl. Thus, besides redirecting carbon flux toward production of $\alpha$-acetolactate through genetic methods, more efficient chemical conversion of $\alpha$-acetolactate into diacetyl should also be developed for optimal production of diacetyl. In the study by Gao et al. ${ }^{12}$, an efficient chemical conversion of $\alpha$-acetolactate to diacetyl could be achieved by addition of $\mathrm{Fe}^{3+}$. However, it was indicated that $\mathrm{Fe}^{3+}$ would also influence the glucose consumption (Figure 4B) and hence might decrease the diacetyl production during the fermentation process. Thus, the addition time of $20 \mathrm{mM} \mathrm{Fe}^{3+}$ was also optimized in the present study. As shown in Figure $4 \mathrm{~A}$, when added at $10 \mathrm{~h}$,
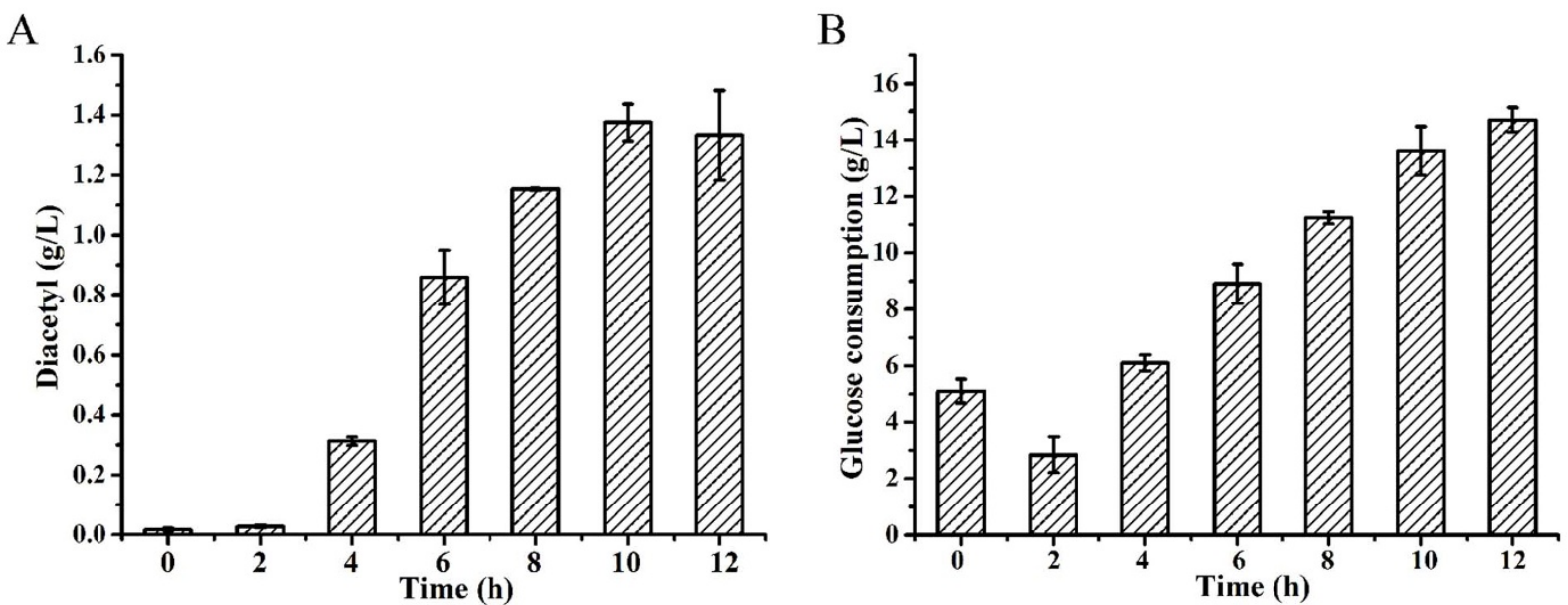

Figure $4 \mid$ Effects of addition time of $\mathrm{Fe}^{3+}$ on diacetyl production (A) and glucose consumption (B). The data were obtained after $14 \mathrm{~h}$ fermentation with $\mathrm{Fe}^{3+}$ addition at different time points. The bioconversion was carried out at $37^{\circ} \mathrm{C}$ in $300-\mathrm{mL}$ shake flasks containing $50 \mathrm{~mL}$ of medium with pH adjusted to 7.4. The final concentration of $\mathrm{Fe}^{3+}$ added to medium was $20 \mathrm{mM}$. Error bars indicate standard deviation $(\mathrm{n}=3)$. 
A

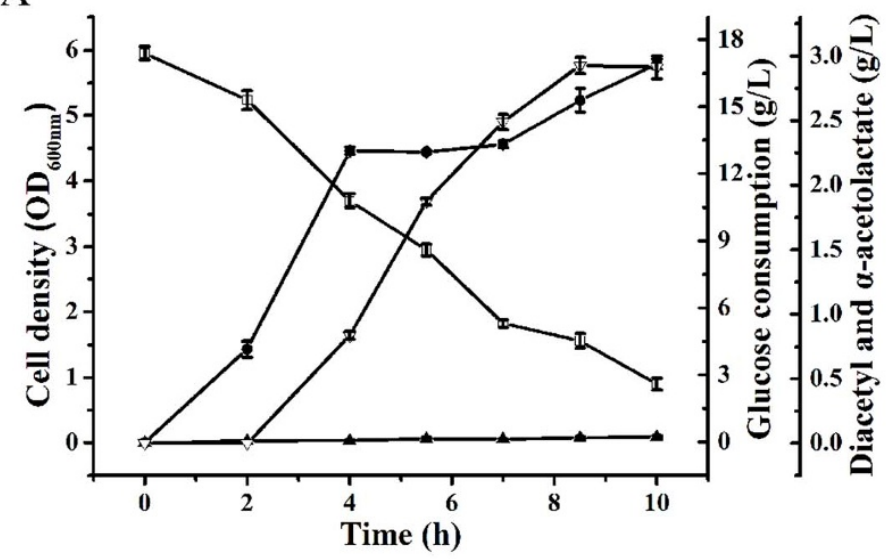

B

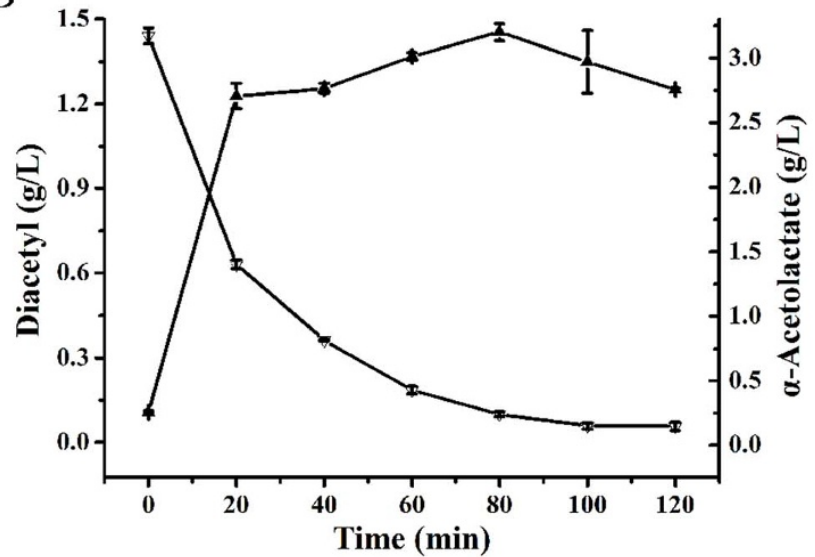

Figure $5 \mid$ Time course of batch fermentation of diacetyl under the optimization condition. Glucose $(\square)$, Cell density $(\bullet), \alpha$-Acetolactate $(\nabla)$, Diacetyl $(\boldsymbol{\Delta})$. (A) Time course of diacetyl and $\alpha$-acetolactate production using E. cloacae SDM $\left(\Delta b u d A \Delta b u d C\right.$ ) before Fe ${ }^{3+}$ addition. (B) Time course of diacetyl production after $20 \mathrm{mM} \mathrm{Fe}^{3+}$ added at $10 \mathrm{~h}$. The bioconversion was carried out at $37^{\circ} \mathrm{C}$ in 300 - $\mathrm{mL}$ shake flasks containing $50 \mathrm{~mL}$ of medium with $\mathrm{pH}$ adjusted to 7.4. The initial glucose concentration used was $17.8 \mathrm{~g} / \mathrm{L}$. Error bars indicate standard deviation $(\mathrm{n}=3)$.

$20 \mathrm{mM} \mathrm{Fe}^{3+}$ could accelerate the NOD of $\alpha$-acetolactate, and accumulate the highest concentration of diacetyl.

Several biotechnological routes have been used to produce diacetyl (Table 2). Among all of the reported biotechnological processes, the group of Liu obtained the highest diacetyl concentration of $4.7 \mathrm{~g} / \mathrm{L}$ with a metabolically engineered C. glabrata ${ }^{12}$. Efforts have been tried in order to increase the yield of diacetyl through inactivation of ALDC and overexpression of NADH oxidase in L. lactis. Using $5 \mathrm{~g} / \mathrm{L}$ glucose as the substrate, the recombinant $L$. lactis produced $0.38 \mathrm{~g} / \mathrm{L}$ diacetyl at a high yield of $0.16 \mathrm{~mol} / \mathrm{mol}$ glucose $^{14}$. In this study, metabolic engineering based on 2,3-BD pathway was used to reconstruct $E$. cloacae SDM as a novel biocatalyst for diacetyl production. Under optimal conditions, the recombinant $E$. cloacae SDM $(\triangle b u d A \Delta b u d C)$ could produce diacetyl with rather high concentration $(1.45 \mathrm{~g} / \mathrm{L})$, productivity $(0.13 \mathrm{~g} /(\mathrm{L} \cdot \mathrm{h}))$ and yield $(0.21 \mathrm{~mol} / \mathrm{mol})$. Both the productivity and yield of diacetyl produced by the recombinant E. cloacae were new records for diacetyl production (Table 2). The carbon flux channeled into the diacetyl biosynthetic might be further enhanced since there were still (3R)-AC, (3S)-AC, and $(2 R, 3 R)-2,3-\mathrm{BD}$ accumulated during the fermentation (Figure $2 \mathrm{IV}$ $\mathrm{C}$ Figure 2 IV-B). This may be accomplished by searching the undiscovered diacetyl reductase, or overexpressing NADH oxidase, which could lead to prevention of NADH dependent reduction of diacetyl.

\section{Methods}

Chemicals and biochemicals. $(2 R, 3 R)-2,3-\mathrm{BD}(98.0 \%),(2 S, 3 S)-2,3-\mathrm{BD}(99.0 \%)$, and meso-2,3-BD (98.0\%) were purchased from ACROS (The Kingdom of Belgium). Racemic AC, ethyl 2-acetoxy-2-methyl-acetoacetate, and diacetyl were purchased from Sigma. NADH was purchased from Amresco. Restriction enzymes were purchased from TaKaRa Bio Inc. (China). PCR primers were prepared by Sangon
(Shanghai, China). FastPfu DNA polymerase and $\mathrm{T}_{4}$ DNA ligase were purchased from Transgen Biotech (China) and MBI (USA), respectively. All other chemicals were of analytical grade and commercially available.

Bacterial strains and plasmids. All the strains and plasmids used in this study are listed in Table 3. E. coli DH5 $\alpha$ was used for general cloning procedures. The pKR6K was used for gene knock-out in E. cloacae strain $\mathrm{SDM}^{24}$. E. coli $\mathrm{S} 17-1$, which is able to host pKR6K and its derivatives, was used for conjugation with E. cloacae SDM. Lysogenic broth (LB) medium was used for the culture of E. coli and E. cloacae SDM. The selection medium in the conjugation experiments was M9 minimal medium supplemented with $1 \%$ sodium citrate as the carbon source and $0.05 \%$ ammonium chloride as the nitrogen source. Solid LB medium with $10 \%$ sucrose was used to select plasmid excision from the chromosome during the gene allelic exchange experiments. Kanamycin was used at a concentration of $50 \mu \mathrm{g} / \mathrm{mL}$.

Knock out of the genes in E. cloacae SDM. Primers used in this study are listed in Table S1. Isolation of vectors, restriction enzyme digestion, agarose gel electrophoresis, and other DNA manipulations are carried out by standard protocols ${ }^{25}$. Mutants of E. cloacae strain SDM were generated by allele exchange using the suicide plasmid $\mathrm{pKR} 6 \mathrm{~K}^{24}$. The left and right flanking sequences were amplified from E. cloacae SDM and then ligated through PCR to get $\triangle b u d A$ fragment using primer pairs P $\Delta b u d A . \mathrm{f}(E c o R I) / \mathrm{P} \Delta b u d A . r$ (overlap), P $\Delta b u d A . f$ (overlap)/P $\Delta b u d A . r$ (BamHI). The gel-purified $\Delta b u d A$ fragments were ligated to the pKR6K vector digested with the EcoRI and BamHI. The resulting plasmid was designated $\mathrm{pK} \Delta b u d A$ For conjugation, donor and recipient strains were grown in LB to initial log phase $\left(\mathrm{OD}_{600 \mathrm{~nm}}=0.5\right)$, then collected and mixed at a ratio of $5: 1$ and spotted on LB plate. After $12 \mathrm{~h}$ of conjugation at $37^{\circ} \mathrm{C}$, cells were recovered by washing the LB plate with normal saline and plated on the selection medium plates to eliminate the donor strain. The merodiploid (single-crossover) genotype was confirmed by PCR using primers P $\Delta b u d A . f(E c o R I)$ and P $\Delta b u d A . r(B a m H I)$. Next, a single merodiploid colony was grown overnight in LB medium and appropriate dilutions were plated onto LB agar with $10 \%(\mathrm{w} / \mathrm{v})$ sucrose, and then incubated overnight at $37^{\circ} \mathrm{C}$. Colonies were screened by PCR using primers P $\Delta b u d A$.f $(E c o R I)$ and P $\Delta b u d A$.r (BamHI). The budC and $g d h$ mutants of strain SDM were generated by the same way of E. cloacae SDM $(\Delta b u d A)$.

Table 2 | Comparison of diacetyl production by different microorganisms

\begin{tabular}{|c|c|c|c|c|c|}
\hline Strain & Engineering strategy & $\begin{array}{l}\text { Diacetyl } \\
(\mathrm{g} / \mathrm{L})\end{array}$ & $\begin{array}{c}\text { Yield } \\
(\mathrm{mol} / \mathrm{mol})\end{array}$ & $\begin{array}{l}\text { Productivity } \\
(\mathrm{g} /(\mathrm{L} \cdot \mathrm{h}))\end{array}$ & Reference \\
\hline L. lactis & Inactivation of ALDC, overexpression of NADH oxidase & 0.38 & 0.16 & 0.03 & 14 \\
\hline L. lactis & Random mutagenesis & 0.52 & $-{ }^{a}$ & 0.02 & 17 \\
\hline E. aerogenes & UV mutation and medium optimization & 1.35 & 0.03 & $--^{\alpha}$ & 16 \\
\hline L. lactis & Inactivation of ALDC, overexpression of NADH oxidase & 0.36 & 0.12 & 0.03 & 18 \\
\hline C. glabrata & $\begin{array}{l}\text { Overexpression of ALS, inactivation of ALDC and DR, medium } \\
\text { optimization }\end{array}$ & 4.70 & 0.10 & 0.07 & 12 \\
\hline
\end{tabular}




\section{Strain}

E. coli $\mathrm{DH} 5 \alpha$

E. coli S17-1

E. cloacae SDM

SDM ( $\triangle$ budA)

SDM ( $\triangle$ budA $\Delta$ budC)

SDM ( $\Delta$ budA $\Delta g d h)$

SDM ( $\Delta$ budA $\Delta$ budC $\Delta$ gdh)

Plasmid

pEASYBlunt

PKR6K

pK $\Delta$ budA

pK $\Delta$ budC

pK $\Delta g d h$

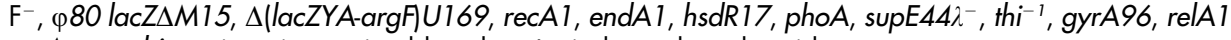
recA, pro, thi, conjugative strain able to host $\lambda$-pir-dependent plasmids

Wild-type

E. cloacae SDM budA disruption mutant strain

E. cloacae SDM budA and budC disruption mutant strain

E. cloacae SDM budA and gdh disruption mutant strain

E. cloacae SDM budA, budC and gdh disruption mutant strain

reference

Novagen

This study

This study

This study

This study

Transgen

24

$\mathrm{Km}^{r}$, gene replacement vector derived from plasmid pK 18 mobsacB, R6K origin, $\mathrm{Mob}^{+} \mathrm{sacB}$

This study

This study

$\mathrm{Km}^{r}$, pKR6K derivative, carries a 587 bp deletion of budA

$\mathrm{Km}^{r}$, pKR6K derivative, carries a $302 \mathrm{bp}$ deletion of $\mathrm{gdh}$

${ }^{A} \mathrm{Ap} r$, ampicilin resistance; $\mathrm{Km}^{r}$, kanamycin resistance.

Batch fermentation. The batch fermentation was conducted in $300-\mathrm{mL}$ shake flasks containing $50 \mathrm{~mL}$ medium. The medium consisted of $\mathrm{M} 9$ medium supplemented with $18 \mathrm{~g} / \mathrm{L}$ glucose and $5 \mathrm{~g} / \mathrm{L}$ yeast extract. The cultivation was carried out at $37^{\circ} \mathrm{C}$ and $180 \mathrm{rpm}$. The initial $\mathrm{pH}$ was adjusted to 7.4. Samples were collected periodically to determine the Cell density, concentrations of glucose, diacetyl, and $\alpha$-acetolactate.

Enzyme activity assays. For the assays of the activities of ALDC and DR, cells of the strain were grown for $8 \mathrm{~h}$, then centrifuged at $13,000 \times \mathrm{g}$ for $5 \mathrm{~min}$, and washed twice with $67 \mathrm{mM}$ phosphate buffer (pH 7.4). Cells were finally resuspended with $67 \mathrm{mM}$ phosphate buffer $(\mathrm{pH} 7.4)$ to an $\mathrm{OD}_{600 \mathrm{~nm}}$ of 20 , and disrupted with an ultrasonic cell breaking apparatus (Xinzhi, Ningbo, China). Cell debris was removed through centrifugation at 13,000 $\times \mathrm{g}$ for $15 \mathrm{~min}$. Enzyme activity was assayed in the resulting supernatant.

The activity of ALDC was assayed by detecting the production of AC from $\alpha$-acetolactate ${ }^{26} . \alpha$-Acetolactate was prepared immediately before use from ethyl 2-acetoxy-2-methyl-acetoacetate according to the protocol supplied by the manufacture. One unit of ALDC activity was defined as the amount of protein that produced $1 \mu \mathrm{mol}$ of AC per min.

The activity of DR was assayed spectrophotometrically by measuring the change in absorbance at $340 \mathrm{~nm}$ corresponding to the oxidation of NADH $\left(\varepsilon_{340}=6,220 \mathrm{M}^{-1}\right.$ $\mathrm{cm}^{-1}$ ) at $30^{\circ} \mathrm{C}$ using a UV/visible spectrophotometer (Ultrospec 2100 pro, Amersham Biosciences, USA $)^{27,28}$. The reaction solution for DR assay contained $5 \mathrm{mM}$ of diacetyl and $0.2 \mathrm{mM}$ of NADH in $67 \mathrm{mM}$ phosphate buffer (pH 7.4). One unit of activity was defined as the amount of enzyme that consumed $1 \mu \mathrm{mol}$ of NADH per min. The protein concentration was measured by the Lowry method, with bovine serum albumin as the standard ${ }^{29}$

Analytical methods. Samples were withdrawn periodically and centrifuged at 12,000 $\times g$ for $10 \mathrm{~min}$. The Cell density was determined by monitoring the absorbance at $600 \mathrm{~nm}$ using a spectrophotometer (LENGGUANG-721, China) after an appropriate dilution. The concentration of glucose was measured enzymatically by a bio-analyzer (SBA-40D, Shandong Academy of Sciences, China) after diluting to an appropriate concentration. The concentrations of 2,3-BD and AC were analyzed by GC as described in $\mathrm{Ma}^{\mathrm{et}} \mathrm{al}^{6}$. The concentrations of $\alpha$-acetolactate and diacetyl were determined by the methods described in the previous reports ${ }^{12,30}$.

1. Celińska, E. \& Grajek, W. Biotechnological production of 2,3-butanediol-current state and prospects. Biotechnol. Adv. 27, 715-725 (2009).

2. Xu, Y. et al. Systematic metabolic engineering of Escherichia coli for high-yield production of fuel bio-chemical 2,3-butanediol. Metab. Eng. 22, 22-33 (2014).

3. Liu, Z. et al. Production of (2S,3S)-2,3-butanediol and (3S)-acetoin from glucose using resting cells of Klebsiella pneumonia and Bacillus subtilis. Bioresour. Technol. 102, 10741-10744 (2011).

4. Ji, X. J., Huang, H. \& Ouyang, P. K. Microbial 2,3-butanediol production: a state of the art review. Biotechnol. Adv. 29, 351-364 (2011).

5. Wang, A. et al. Efficient 2,3-butanediol production from cassava powder by a crop-biomass-utilizer, Enterobacter cloacae subsp. dissolvens SDM. PLoS One 7, e40442 (2012)

6. Ma, C. et al. Enhanced 2,3-butanediol production by Klebsiella pneumoniae SDM. Appl. Microbiol. Biotechnol. 82, 49-57 (2009).

7. Li, L. et al. A newly isolated Bacillus licheniformis strain thermophilically produces 2,3-butanediol, a platform and fuel bio-chemical. Biotechnol. Biofuels 6, 123 (2013).

8. Zhang, L. et al. Microbial production of 2,3-butanediol by a mutagenized strain of Serratia marcescens H30. Bioresour. Technol. 101, 1961-1967 (2010).
9. Ji, X. J. et al. Cofactor engineering through heterologous expression of an NADH oxidase and its impact on metabolic flux redistribution in Klebsiella pneumoniae. Biotechnol. Biofuels 6, 7 (2013).

10. Oliver, J. W., Machado, I. M., Yoneda, H. \& Atsumi, S. Combinatorial optimization of cyanobacterial 2,3-butanediol production. Metab. Eng. 22, 76-82 (2014)

11. Chen, C., Wei, D., Shi, J., Wang, M. \& Hao, J. Mechanism of 2,3-butanediol stereoisomer formation in Klebsiella pneumoniae. Appl. Microbiol. Biotechnol. 98, 4603-4613 (2014).

12. Gao, X., Xu, N., Li, S. \& Liu, L. Metabolic engineering of Candida glabrata for diacetyl production. PLoS One 9, e89854 (2014).

13. Aymes, F., Monnet, C. \& Corrieu, G. Effect of $\alpha$-acetolactate decarboxylase inactivation on $\alpha$-acetolactate and diacetyl production by Lactococcus lactis subsp. lactis biovar diacetylactis. J. Biosci. Bioeng. 87, 87-92 (1999).

14. Hugenholtz, J. et al. Lactococcus lactis as a cell factory for high-level diacetyl production. Appl. Environ. Microbiol. 66, 4112-4114 (2000).

15. Xu, P., Hua, D. \& Ma, C. Microbial transformation of propenylbenzenes for natural flavour production. Trends Biotechnol. 25, 571-576 (2007).

16. Zhao, L., Bao, Y., Wang, J., Liu, B. \& An, L. Optimization and mechanism of diacetyl accumulation by Enterobacter aerogenes mutant UV-3. World J. Microbiol. Biotechnol. 25, 57-64 (2009).

17. Monnet, C., Aymes, F. \& Corrieu, G. Diacetyl and $\alpha$-acetolactate overproduction by Lactococcus lactis subsp. lactis biovar diacetylactis mutants that are deficient in $\alpha$-acetolactate decarboxylase and have a low lactate dehydrogenase activity. Appl. Environ. Microbiol. 66, 5518-5520 (2000).

18. Guo, T., Kong, J., Zhang, L., Zhang, C. \& Hu, S. Fine tuning of the lactate and diacetyl production through promoter engineering in Lactococcus lactis. PLoS One 7, e36296 (2012)

19. Jyoti, B. D., Suresh, A. K. \& Venkatesh, K. V. Diacetyl production and growth of Lactobacillus rhamnosus on multiple substrates. World J. Microbiol. Biotechnol. 19, 509-514 (2003)

20. Hugenholtz, J. \& Starrenburg, M. Diacetyl production by different strains of Lactococcus lactis subsp. lactis var. diacetylactis and Leuconostoc spp. Appl. Microbiol. Biotechnol. 38, 17-22 (1992).

21. Xu, Y. et al. Genome sequence of Enterobacter cloacae subsp. dissolvens SDM, an efficient biomass-utilizing producer of platform chemical 2,3-butanediol. J. Bacteriol. 194, 897-898 (2012).

22. Howard-Flanders, P. \& Theriot, L. Mutants of Escherichia coli K-12 defective in DNA repair and in genetic recombination. Genetics. 53, 1137-1150 (1966).

23. Mohr, B., Aymes, F., Rea, M. C., Monnet, C. \& Cogan, T. M. A new method for the determination of 2-acetolactate in dairy products. Int. Dairy. J. 7, 701-706 (1997).

24. Wang, Y., Tao, F. \& Xu, P. Glycerol dehydrogenase plays a dual role in glycerol metabolism and 2,3-butanediol formation in Klebsiella pneumoniae. J. Biol. Chem. 289, 6080-6090 (2014).

25. Sambrook, J. \& Russel, D. Molecular Cloning: A Laboratory Manual. Third edn, (Cold Spring Harbor Laboratory Press, Cold Spring Harbor, NY, 2001).

26. Phalip, V. et al. Purification and properties of the $\alpha$-acetolactate decarboxylase from Lactococcus lactis subsp. lactis NCDO 2118. FEBS. Lett. 351, 95-99 (1994).

27. Zhang, L. et al. Cloning, expression and characterization of glycerol dehydrogenase involved in 2,3-butanediol formation in Serratia marcescens H30. J. Ind. Microbiol. Biotechnol. doi:10.1007/s10295-014-1472-x (2014).

28. Zhang, L. et al. A new $\mathrm{NAD}(\mathrm{H})$-dependent meso-2,3-butanediol dehydrogenase from an industrially potential strain Serratia marcescens H30. Appl. Microbiol. Biotechnol. 98, 1175-1184 (2014).

29. Hartree, E. F. Determination of protein: a modification of the Lowry method that gives a linear photometric response. Anal. Biochem. 48, 422-427 (1972). 
30. Benson, K. H., Godon, J., Renault, P., Griffin, H. G. \& Gasson, M. J. Effect of ilvBNencoded $\alpha$-acetolactate synthase expression on diacetyl production in Lactococcus lactis. Appl. Microbiol. Biotechnol. 45, 107-111 (1996).

31. Simon, R., Priefer, U. \& Pühler, A. A broad host range mobilization system or in vivo genetic engineering: transposon mutagenesis in gram negative bacteria. Nat. Biotechnol. 1, 784-791 (1983).

\section{Acknowledgments}

This work was supported by Chinese National Program for High Technology Research and Development (2011AA02A207 and 2012AA022104), the National Natural Science Foundation of China (31470164 and J1103515), the Excellent Middle-Aged and Youth Scientist Award Foundation of Shandong Province (BS2013SW025), and the Program for High Technology Research and Development of Shandong Province (2014GSF121030).

\section{Author contributions}

C.G. and C.M. participated in the design of the study. L.Z., Y.Z., Q.L. and L.M. executed the experimental work. L.Z., M.H., K.L. and M.L. analyzed the data. C.G., C.M. and P.X. contributed reagents and materials. L.Z., C.G., C.M. and P.X. wrote and revised the manuscript. All authors read and approved the final manuscript.

\section{Additional information}

Supplementary information accompanies this paper at http://www.nature.com/ scientificreports

Competing financial interests: The authors declare no competing financial interests.

How to cite this article: Zhang, L. et al. Production of diacetyl by metabolically engineered Enterobacter cloacae. Sci. Rep. 5, 9033; DOI:10.1038/srep09033 (2015).

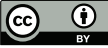

This work is licensed under a Creative Commons Attribution 4.0 International License. The images or other third party material in this article are included in the article's Creative Commons license, unless indicated otherwise in the credit line; if the material is not included under the Creative Commons license, users will need to obtain permission from the license holder in order to reproduce the material. To view a copy of this license, visit http://creativecommons.org/licenses/by/4.0/ 